\title{
Editorial
}

\section{Responding to the requirements of the National Service Framework for coronary disease: a core data set for myocardial infarction}

Clinical audit - the systematic assessment and improvement of the quality of care-is now an essential requirement of all clinicians by the government. Clinical audit of the process of myocardial infarction has been performed both in this country and elsewhere for several years, ${ }^{1-3}$ although the majority of work is performed within individual hospitals rather than in collaboration with colleagues in other hospitals. It has tended to concentrate on the use of thrombolytic treatment. Recent evidence from 15 hospitals examined between 1993 and 1997 showed only patchy improvement in delays from a call for help to treatment. Some hospitals where there were long delays before thrombolytic treatment showed no improvement over that time. ${ }^{4}$ It was clear from these data that in some hospitals either the incentive or the facilities to improve performance, at least in this area, was lacking.

The National Service Framework for Coronary Heart Disease, ${ }^{5}$ has been compiled with advice from cardiologists and others involved with the management of coronary heart disease. It requires annual clinical audit of a number of aspects of the management of myocardial infarction. It sets out explicit targets to be achieved in areas such as the delays between a call for help and access to a defibrillator and reperfusion treatment. An annual audit of the prescription of $\beta$ blockers, aspirin, and lipid lowering treatment for the secondary prevention of infarction is also required. In time 30 day mortality data will have to be collected for patients with myocardial infarction who are admitted to hospital. Setting these standards may be the easier part of the exercise; achieving them and monitoring practice throughout the country will be a greater challenge. It will be crucial that the data collected to satisfy these requirements are credible. Clinicians will need to be confident that data supplied by their units are accurate, and that subsequent analysis is valid, taking account of case mix variables and age, in order to provide meaningful information.

Clinical data collection and analysis by the National Health Service (NHS) has, however, long been a subject of concern, and recent publication of mortality tables for acute myocardial infarction has done little to dispel concern by clinicians that the data by which they are judged may be flawed. ${ }^{6}$ Norris and his colleagues in the Southern Heart Attack Response (SHARP) group have shown that the sensitivity of hospital clinical coding to identify cases of myocardial infarction which were identified by conventional criteria in the SHARP study was only $80 \%$, and the positive predictive value of a diagnosis of infarction made by clinical coding was $87 \%$ (RM Norris, unpublished data, 1999). A disparity has also been demonstrated between estimates of coronary mortality made according to strict clinical and pathological definitions and the official rates which are based on death certification. In the age band 65-74 years there was a shortfall of about $20 \%$ in deaths certified by strict criteria compared with those having myocardial infarction entered as the pri- mary cause of death. ${ }^{7}$ From 75 years of age and upwards, wherein the majority of deaths from coronary disease are recorded, the disparity may be even greater. This is due to the use of a diagnosis of myocardial infarction on death certificates without confirmatory evidence. Both these general factors distort mortality data and other analyses which take no account of these deficiencies. Both need to be addressed in order to provide credible data.

A further difficulty in comparing performance of hospitals has been a lack of simple definitions with which to define the standards which now need to be measured. This has now been made easier by the development of a core data set for myocardial infarction by a group working under the aegis of the Royal College of Physicians, and the British Cardiac Society. ${ }^{8}$ This sets out definitions for groups of fields covering demography, pre-hospital and in-hospital delays before treatment, other aspects of hospital care, cardiac arrest and resuscitation, secondary prevention measures, and the use of investigations and interventions. Each field is provided with definitions, evidence justifying inclusion, and a separate commentary on the use of each field, identifying confounders and other practical issues related to their use.

A proposal to use the data set to allow hospitals to examine their performance in achieving standards for the management of myocardial infarction set out in the National Service Framework has now been funded by the Department of Health. Good quality audit is hard work and time consuming; this proposal will allow clinicians to engage in national audit, and for their hospitals to satisfy the requirements of the National Service Framework, in as simple a manner as possible. Locally collected data will be recorded in a computer database using the core data set definitions, and accessed via the NHS net or by modem by a central server run by the Central Cardiac Audit Database group. ${ }^{9}$ The security and confidentiality of data are paramount and have received close attention. Data analysis will be performed centrally and analyses immediately returned to clinicians with comparative data from other hospitals. Clinicians and managers will then be able to assess their performance in the light of comparable national data. After a pilot phase it is proposed that this audit will be extended to cover all acute hospitals in England in less than two years. This will be the first practical use of the data set, although the data set can be used, in whole or in part, for other clinical or audit studies of myocardial infarction where collaboration between hospitals is required.

This will be an opportunity to demonstrate that high quality data can be collected from hospitals and analysed appropriately. Clinicians should be able to accept the resulting analyses as a fair reflection of the quality of care offered to their patients with myocardial infarction. These data will also be available to local managers and in suitable form to the Department of Health. In aggregated form they will in time provide high quality longitudinal national data on the 
management of myocardial infarction. As a first priority they should be used to demonstrate that we offer high quality clinical services for patients with myocardial infarction, and that these are evenly distributed across the country.

J S BIRKHEAD

Department of Cardiology,

Northampton General Hospital,

Northampton NN1 5BD, UK

e mail:john.birkhead@NGH-tr.anglox.nhs.uk

1 Birkhead JS, on behalf of the Myocardial Infarction Audit Group. Thrombolytic treatment for myocardial infarction: an examination of practice in 39 United Kingdom hospitals. Heart 1997;78:28-33.

2 Lambrew CT, Bowlby LJ, Rogers WJ, et al. Factors influencing the time to thrombolysis in acute myocardial infarction. Time to thrombolysis substudy of the national registry of myocardial infarction-1. Arch Intern Med 1997;157:2577-82.
3 Rustige J, Schiele R, Burzyk U, et al. The 60 minutes myocardial infarction project. Treatment and outcome of patients with acute myocardial infarction in Germany. Eur Heart $\mathcal{F}$ 1997;18:1438-46.

4 Birkhead JS, on behalf of the Myocardial Infarction Audit Group. Trends in the provision of thrombolytic treatment 1993-1997. Heart 1999;82:43842 .

5 Anon. National Service Framework for Coronary Heart Disease. Modern standards and service models. London: Department of Health, March 2000.

6 Anon. Quality and performance in the NHS: clinical indicators, section 4.3. London: NHS Executive, Department of Health, June 1999.

7 United Kingdom Heart Attack Study Collaborative Group. The falling mortality from coronary heart disease: a clinicopathological perspective. The United Kingdom heart attack study (UKHAS) collaborative group. Heart 1998;80:121-6.

8 Birkhead JS, Norris RM, Quinn T, et al, on behalf of the Coronary Heart Disease National Service Framework Steering Group. Acute myocardial infarction: a core data set for monitoring standards of care. London: Royal College of Physicians, December 1999.

9 Rickards A, Cunningham D. From quantity to quality; the central cardiac audit database. Heart 1999;82 (suppl II):II18-22.

\section{IMAGES IN CARDIOLOGY}

\section{Myocardial haemangioma: echocardiographic, MRI, and anatomical correlation}

A 44 year old woman presented with intermittent atypical chest pain and epigastric discomfort of three months' duration. An ECG revealed anterior $\mathrm{T}$ wave inversion (leads V2-V6). A chest radiograph was normal. Transthoracic echocardiography revealed a mass in the distal inferoposterior wall of the left ventricle. A transoesophageal echocardiogram was performed. This confirmed a well defined mass measuring $4.0 \times 3.5 \mathrm{~cm}$ with a heterogenous echotexture and apparent small cystic cavities in the distal posteroinferior wall of the left ventricle. There was no evidence of pericardial involvement. Magnetic resonance imaging (MRI) revealed a well defined, solid $4.8 \times 4.3 \mathrm{~cm}$ mass arising from the apical posteroinferior left ventricular myocardium, with a mildly heterogenous signal on T1, a very bright signal on STIR, and pronounced contrast enhancement (left). This was felt to be a benign vascular tumour such as a haemangioma. A blood screen, including hydatid serology, and an abdominal ultrasound scan were normal.

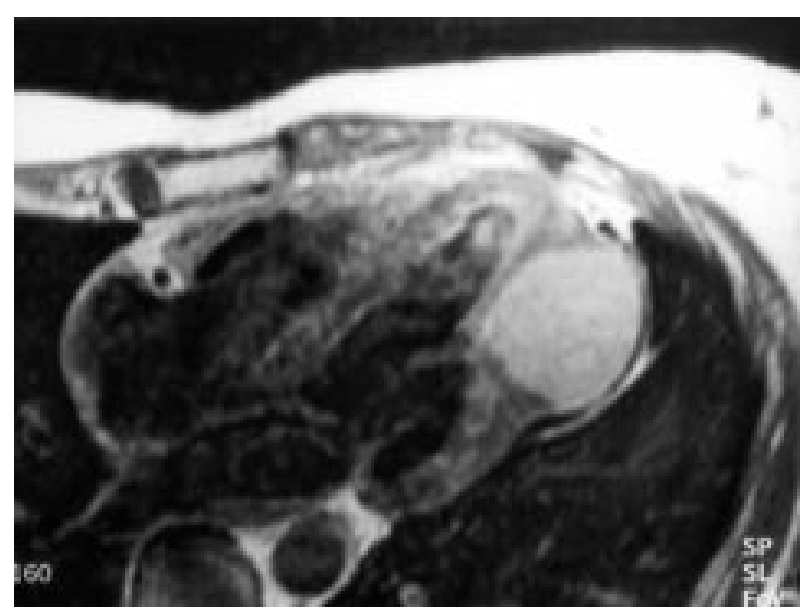

A transarterial biopsy was considered but as the tumour did not extend to the endocardium and there was no infiltration into the pericardium, the decision was made to perform a complete excision biopsy with cardiopulmonary bypass. The surgical specimen measured $5.1 \times 3.6 \mathrm{~cm}$, and had the appearances of a benign haemangioma (right) which was confirmed on subsequent histology. It was completely excised and the myocardium sutured without the need for a patch. The base of the posterior papillary muscle required reinforcement with pledgetted ticron sutures. A postoperative transthoracic echocardiogram showed good left ventricular function with only mild mitral regurgitation. The patient made an uneventful recovery and was discharged from hospital on the sixth postoperative day.

PETER N RUYGROK

CHRISTOPHER J OCCLESHAW MALCOLM E LEGGET ALAN R KERR

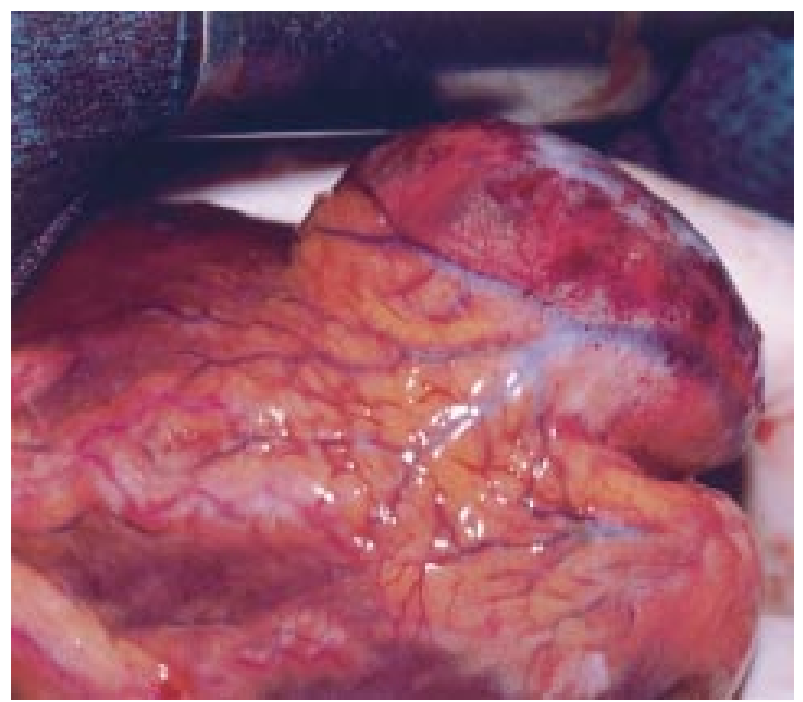

